\title{
Effect of dispersion interactions on the properties of LiF in condensed phases
}

\author{
Dario Corradini ${ }^{1,2}$, Dario Marrocchelli ${ }^{3}$, Paul A. Madden ${ }^{4}$ and Mathieu Salanne ${ }^{1,2}$ \\ 1 Sorbonne Universités, UPMC Univ Paris 06, UMR 8234, PHENIX, F-75005, Paris, France \\ 2 CNRS, UMR 8234, PHENIX, F-75005, Paris, France \\ 3 Department of Materials Science and Engineering, \\ Massachusetts Institute of Technology, Cambridge, MA, USA and \\ 4 Department of Materials, University of Oxford, Parks Road, Oxford OX1 3PH, UK
}

\begin{abstract}
Classical molecular dynamics simulations are performed on $\mathrm{LiF}$ in the framework of the polarizable ion model. The overlap-repulsion and polarization terms of the interaction potential are derived on a purely non empirical, first-principles basis. For the dispersion, three cases are considered: a first one in which the dispersion parameters are set to zero and two others in which they are included, with different parameterizations. Various thermodynamic, structural and dynamic properties are calculated for the solid and liquid phases. The melting temperature is also obtained by direct coexistence simulations of the liquid and solid phases. Dispersion interactions appear to have an important effect on the density of both phases and on the melting point, although the liquid properties are not affected when simulations are performed in the $N V T$ ensemble at the experimental density.
\end{abstract}

\section{INTRODUCTION}

Dispersion interactions between two chemical entities arise from the instantaneous correlation of the fluctuations of their electron densities [1]. They are the only attractive interactions in noble gases and are therefore at the origin of the existence of their pure condensed phases [2]. But dispersion interactions are generally weaker than the other intermolecular interactions in molecular systems and their role in systems for which stronger attractive forces are present is hard to establish.

In density functional theory (DFT) calculations, dispersion interactions are difficult to capture. Several methods have been developed, in which they are treated directly by devising new functionals [3, 4] or effectively by adding an analytical term to existing functionals [5]. Following these developments, it was shown that dispersion interactions impact structure and dynamics in a variety of systems; for example in water at ambient 6 or supercritical conditions 7. But the most affected quantity is usually the density, which is understimated by as much as $20 \%$ in water when dispersion interactions are not accounted for [8]. Similar deviations are found in crystalline systems [9]. In ionic materials, the effect of dispersion is expected to be smaller due to the presence of a strong attractive Coulombic interaction between species with different charges. This leads to strong charge ordering effects, and the overlap-repulsion interaction sets the distance of closest approach between two ions. The polarization (induction) interaction also plays a well-identified role and it is at the origin of the stabilization of non-trivial crystal structures and of the crosslinking of the coordination shells of multivalent cations in ionic melts [10. Nevertheless, it was shown by Kirchner et al. that in "room-temperature" ionic liquids, the inclusion of dispersion interactions in DFT calculations modifies the vibrational and dynamic properties of the system $[11-13]$.
DFT-based molecular dynamics is however limited to short simulation times and small system sizes, so that it is hard to establish clearly the role of dispersion on a wide range of properties. Classical molecular dynamics is an alternative option, provided that the force field accurately separates the various contributions from Coulombic, repulsion, polarization and dispersion interactions. In recent years, we have developed a series of methods which make possible the derivation of such force fields for inorganic ionic materials 14 17. In short, we use a polarizable ion model, and all the parameters except the ones concerning dispersion are fitted to standard condensed phase DFT calculations. The emphasis on "condensed phase" here is because the physical properties of ions are strongly affected by their coordination environment and the use of $a b$ initio data on isolated ions or on the interaction energies of small clusters leads to hopelessly inadequate force-fields. This applies particularly to the dispersion coefficients (and polarizabilities). For our procedure the reference data includes both the dipole moments on each ion and the total force which is exerted on them, allowing the polarization and the repulsion terms to be fitted independently (formal charges are used for the ions, which automatically sets the Coulombic interaction). In addition, some parameters such as the condensed phase polarizability of the ions can directly be calculated 18 20. The dispersion coefficients have to be treated separately, because on one hand the dispersion effects are not yet reliably represented in the DFT calculations and on the other they contribute relatively weakly to the forces on the ions, which means that they do not strongly affect the quality of the fit to the DFT data with the classical force-field.

In the present work, we perform simulations of $\mathrm{LiF}$ in the crystal and liquid phases. We compare the thermodynamic, structural and dynamic properties obtained with two different sets of condensed phase dispersion coefficients, obtained either from the Coupled Hartree- 
Fock (CHF) theory [21] or from a recent method [16, 22] involving the use of the Maximally Localized Wannier Functions (MLWFs) 23, 24 in DFT, as well as with the case where the dispersion interactions are set to zero. LiF was chosen because it is a material for which we expect that the effect of the dispersion interactions is among the lowest. Dispersion is negligible for the $\mathrm{Li}^{+}$cation due to its small radius. Also, $\mathrm{F}^{-}$is the halide anion with the smallest polarizability.

\section{NUMERICAL METHODS}

\section{Polarizable Ion Model}

The polarizable ion model includes Coulombic, dispersion, overlap repulsion and polarization components [25]. First the Coulombic term is:

$$
V^{\text {Coul }}=\sum_{i<j} \frac{q^{i} q^{j}}{r^{i j}}
$$

where $q^{i}$ is the charge on ion $i$, and formal charges are used throughout. The dispersion component includes dipole-dipole and dipole-quadrupole terms

$$
V^{\mathrm{disp}}=-\sum_{i<j}\left(f_{6}^{i j}\left(r^{i j}\right) \frac{C_{6}^{i j}}{\left(r^{i j}\right)^{6}}+f_{8}^{i j}\left(r^{i j}\right) \frac{C_{8}^{i j}}{\left(r^{i j}\right)^{8}}\right)
$$

where $C_{6}^{i j}\left(C_{8}^{i j}\right)$ is the dipole-dipole (dipole-quadrupole) dispersion coefficient, and $f_{n}^{i j}$ are damping functions [26], describing the short-range penetration correction to the asymptotic multipole expansion of dispersion 27. $\left(f_{n}^{i j}(0)=0\right.$ and $\left.f_{n}^{i j}(\infty)=1\right)$. They take the form

$$
f_{n}^{i j}\left(r^{i j}\right)=1-\mathrm{e}^{-b_{n}^{i j} r^{i j}} \sum_{k=0}^{n} \frac{\left(b_{n}^{i j} r^{i j}\right)^{k}}{k !}
$$

and the parameters $b_{n}^{i j}$ represent the distance at which the correction begins to be taken into account. The repulsion overlap component is given by

$$
V^{\mathrm{rep}}=\sum_{i<j} B^{i j} \mathrm{e}^{-a^{i j} r^{i j}}
$$

Finally the polarization part of the potential includes charge-dipole and dipole-dipole terms:

$$
\begin{aligned}
V^{\mathrm{pol}}= & \sum_{i<j}\left(q^{i} \mu_{\alpha}^{j} g_{D}^{i j}\left(r^{i j}\right)-q^{j} \mu_{\alpha}^{i} g_{D}^{j i}\left(r^{i j}\right)\right) \mathbb{T}_{\alpha}^{(1)} \\
& -\sum_{i<j} \mu_{\alpha}^{i} \mu_{\beta}^{j} \mathbb{T}_{\alpha \beta}^{(2)}+\sum_{i} \frac{1}{2 \alpha^{i}}\left|\vec{\mu}^{i}\right|^{2}
\end{aligned}
$$

Here $\mathbb{T}_{\alpha}^{(1)}$ and $\mathbb{T}_{\alpha \beta}^{(2)}$ are the charge-dipole and dipoledipole interaction tensors and $\alpha^{i}$ is the polarizability of

\begin{tabular}{|l|c|c|c|c|}
\hline Ion pair & $A^{i j}$ & $a^{i j}$ & $b_{D}^{i j}=b_{D}^{j i}$ & $c_{D}^{i j}$ \\
\hline $\mathrm{F}^{-}-\mathrm{F}^{-}$ & 282.3 & 2.444 & - & - \\
$\mathrm{F}^{-}-\mathrm{Li}^{+}$ & 18.8 & 1.947 & 1.834 & 1.335 \\
$\mathrm{Li}^{+}-\mathrm{Li}^{+}$ & 1.0 & 5.0 & - & - \\
\hline
\end{tabular}

TABLE I: Fitted parameters for the repulsion and polarization terms for LiF (atomic units). The fluoride polarizability was set to 7.9 atomic units, and the $\mathrm{Li}^{+}$is not polarizable.

ion $i$. Again, we include some short-range effects which are due to the high compression of the ions in condensed ionic materials 28 30. These short-range induction effects are straightforwardly included through the use of damping functions similar to the ones used in the dispersion term:

$$
g_{D}^{i j}\left(r^{i j}\right)=1-c_{D}^{i j} \mathrm{e}^{-b_{D}^{i j} r^{i j}} \sum_{k=0}^{4} \frac{\left(b_{D}^{i j} r^{i j}\right)^{k}}{k !} .
$$

Here $c_{D}^{i j}$ is a parameter that reflects the amplitude of this damping at ion $j$ due to the presence of $i$ and $b_{D}^{i j}$ again is a range parameter.

The instantaneous values of the dipole moments $\left\{\vec{\mu}^{i}\right\}_{N}$ are obtained by minimization of $V^{\text {pol }}$ with respect to these variables: they will therefore depend on the instantaneous positions of neighboring ions and consequently change at each timestep in an MD run. The interaction potential can therefore be seen to contain three additional degrees of freedom (induced dipoles), which describe the state of the electron charge density of the ions. When calculating the forces on the ions in an MD simulation, these electronic degrees of freedom should have their "Born-Oppenheimer" values, which minimize the total potential energy, for every atomic configuration. We search for the ground state configurations of these degrees of freedom at each time step, using a conjugate gradient routine 31. The dynamics is thus similar to the so-called Born-Oppenheimer first-principles molecular dynamics, as implemented, for example, in the CP2K code 32 .

We perform an Ewald summation of all electrostatic interactions and also of dispersion [33. Thus, all those interactions are free from truncation errors. The shortrange repulsion, which is an exponentially decaying function of distance, is however truncated beyond a distance equal to, at maximum, half the shortest dimension of the simulation cell.

\section{Parameterization}

Parameters for the repulsion and polarization terms were obtained from a generalized force-fitting procedure described elsewhere 14, 17. This approach has been 


\begin{tabular}{|l|c|c|}
\hline Setup & $C_{6}$ & $C_{8}$ \\
\hline No dispersion & 0.0 & 0.0 \\
CHF & 15.0 & 150.0 \\
Wannier & 26.3 & 87.7 \\
\hline
\end{tabular}

TABLE II: $\mathrm{F}^{-}-\mathrm{F}^{-}$dispersion parameters for the three simulation setups (atomic units). $b_{6}^{i j}=b_{8}^{i j}=1.9$, and the other $C_{6}$ and $C_{8}$ coefficients (for $\mathrm{Li}^{+}-\mathrm{Li}^{+}$and $\mathrm{Li}^{+}-\mathrm{F}^{-}$) are set to zero in all cases.

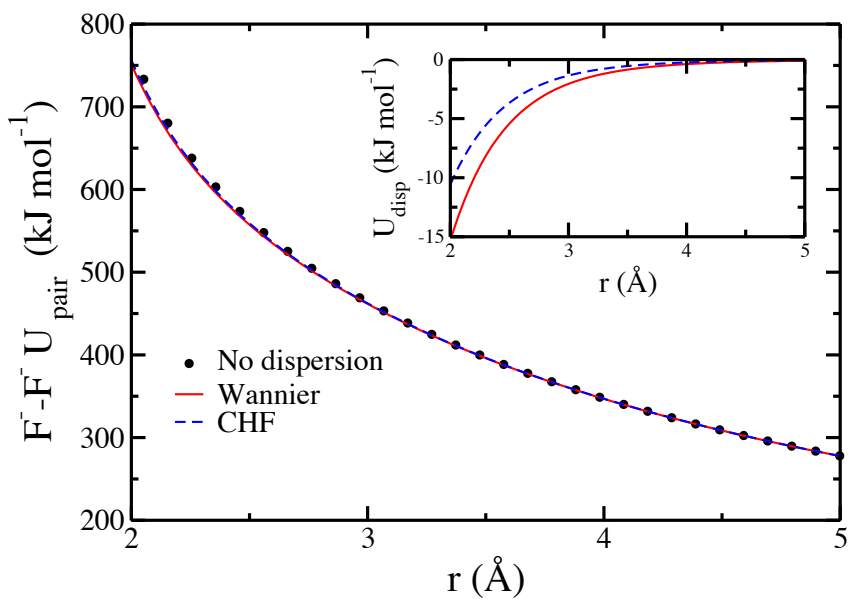

FIG. 1: Sum of the three pairwise additive terms for the $\mathrm{F}^{-}$$\mathrm{F}^{-}$interaction (inset: dispersion term only). The distance range corresponds to the extent of the first peak of the F-F partial radial distribution function in liquid $\mathrm{LiF}$ (shown in Figure 2.

used successfully for a series of oxide and fluoride materials [34 38]. Note that the PBE functional was used to provide the reference data to which the parameters were fitted [39]. From our experience, this functional can be considered as "dispersion-free" in the case of ionic materials. The obtained parameters are provided in Table I. As for the dispersion term, three different cases are tested. In the first case considered, we set it to zero. In the second one ("CHF"), we have taken $C_{6}$ and $C_{8}$ parameters which reproduce the Coupled Hartree-Fock calculation by Fowler et al. 21. Finally, in the third case ("Wannier"), these parameters were determined from a calculation of the MLWFs [23, 24] using the procedure described in references [16, 17. The three sets of dispersion coefficients are provided in Table II

The only interaction which differs between the different cases is the $\mathrm{F}^{-}-\mathrm{F}^{-}$one. The sum of the three pairwise additive terms is plotted for each parameters set in Figure 1, in the region corresponding to first neighbour typical distances. The inset shows the dispersion term only. It appears obvious that the dispersion term only provides a tiny contribution (between 10 and $15 \mathrm{~kJ}$ $\mathrm{mol}^{-1}$ for the shortest distance) to the total interaction potential (which takes values ranging between 450 and $750 \mathrm{~kJ} \mathrm{~mol}^{-1}$ in the same region).

\section{Simulation details}

We consider the rocksalt (B1) $8 \times 8 \times 8$ (2048 LiF units) crystal structure for the solid phase. We build the crystal using the $\mathrm{LiF}$ lattice parameter at ambient conditions, $a=4.026 \AA$. The solid thus obtained is equilibrated in the NPT ensemble [40, at $T=300 \mathrm{~K}$ and ambient pressure, using a thermostat time constant $\tau_{T}=1 \mathrm{ps}$ and an isotropic barostat time constant $\tau_{P}=2 \mathrm{ps}$. Equilibrium is rapidly reached over 10 ps simulations. We then simulate the crystal for accumulating the values of the density over another $20 \mathrm{ps}$.

For the liquid phase, we do two series of simulations. On the one hand we perform $N V T$ runs at $T=1200 \mathrm{~K}$ and at the experimental density, at ambient pressure, $\rho_{\exp }=1.77 \mathrm{~g} \mathrm{~cm}^{-3}$ 41]. The simulation cell for the liquid phase contains $500 \mathrm{LiF}$ units. The temperature is controlled by a Nosé-Hoover thermostat chain [42, 43] with a time-constant $\tau_{T}=10 \mathrm{ps}$.

On the other hand we also perform NPT simulations at $T=1200 \mathrm{~K}$ and at ambient pressure in order to determine the equilibrium density produced by each of the three sets of parameters. In this case we use $432 \mathrm{LiF}$ units. The pressure is controlled by applying a barostat [40, with $\tau_{P}$ and $\tau_{T}$ set to $10 \mathrm{ps.}$

The melting point calculations are performed by direct simulations of the coexisting crystalline and molten phase in the NPT ensemble [44-46]. The simulation cell contains $896 \mathrm{LiF}$ units and it has a large aspect ratio, initially $L_{x}=L_{y}=L_{z} / 4$. First we perform $N V E$ simulations that allow us to roughly estimate the melting temperature as described in Ref. [47. Starting from the final ionic configurations from the $N V E$ runs, which contain a crystalline and liquid region close to coexistence, we then proceed to perform NPT direct coexistence simulations at ambient pressure and at several temperatures in proximity of the melting temperature estimated by the $N V E$ method. The thermostat time constant is set to $1 \mathrm{ps}$ and the anisotropic barostat time constant is set to 2 ps. The angles between the cell vectors are fixed to $\pi / 2$, so that the symmetry of the cell remains orthogonal, but the lengths of the sides of the simulation cell are able to fluctuate independently in order that the calculated pressure tensor is, on average, isotropic with the diagonal elements equal to ambient pressure. The time step for the integration of the equations of motion is $1 \mathrm{fs}$. As the simulation progresses, we monitor the extent of the crystalline and liquid regions over a 500 ps run. For temperature significantly above or below $T_{m}$, the whole cell tends towards becoming molten or crystalline and this enables us to identify a temperature at which it is possible to conduct a run at which the extent of the liq- 


\begin{tabular}{|l|c|c|}
\hline Setup & $\rho^{\text {sol }}\left(\mathrm{g} \mathrm{cm}^{-3}\right)$ & $\rho^{\text {liq }}\left(\mathrm{g} \mathrm{cm}^{-3}\right)$ \\
\hline Experiment & 2.64 & 1.77 \\
No dispersion & 2.32 & 1.50 \\
CHF & 2.38 & 1.62 \\
Wannier & 2.42 & 1.69 \\
\hline
\end{tabular}

TABLE III: Equilibrium densities of solid LiF at $T=300 \mathrm{~K}$ and of liquid $\mathrm{LiF}$ at $T=1200 \mathrm{~K}$.

uid and solid regions can coexist over the length of the simulation.

\section{RESULTS}

\section{Density}

Equilibrium densities extracted from the NPT simulations of solid and liquid $\mathrm{LiF}$ at respective temperatures of 300 and $1200 \mathrm{~K}$ are provided in Table III. The comparison with experiments shows that similar differences are obtained in both cases when dispersion effects are included. The largest difference is observed when they are omitted, with an underestimation of $15.3 \%$ for $\rho^{\text {liq }}$. Such a deviation is of the same order of magnitude as that obtained in liquid water at room-temperature from DFT calculations using PBE or BLYP functionals [8]. When the dispersion effects are included, the liquid density remains underestimated, by $4.5 \%$ and $8.5 \%$ in the Wannier and CHF cases, respectively. We note that in order to reproduce more accurately the experimental density, larger values would be necessary for $C_{6}$ and $C_{8}$ parameters. Such an underestimation of the density was already observed in our previous work on $\mathrm{LiF}_{-} \mathrm{ThF}_{4}$, while a perfect agreement was obtained for LiF-NaF-KF and $\mathrm{NaF}_{-} \mathrm{ZrF}_{4}$ mixtures [4, 49] (both studies used the CHF parameters). This shows that although the polarizable ion model transferability for multiple physical and chemical conditions is well established, a completely transferable model would require more complex functional form, in which "environmental effects" are taken into account. These environmental effects have mainly been studied for the polarizability [50, 51, and since dispersion effects follow a similar dependence on the electronic cloud extension as does the former, equivalent functional forms could be employed.

\section{Liquid properties at fixed density}

In liquid water, the dispersion interaction was shown to impact strongly the structure and dynamics at ambient [6] and supercritical [7] conditions, even at fixed density. In particular, the first peaks of the O-O and

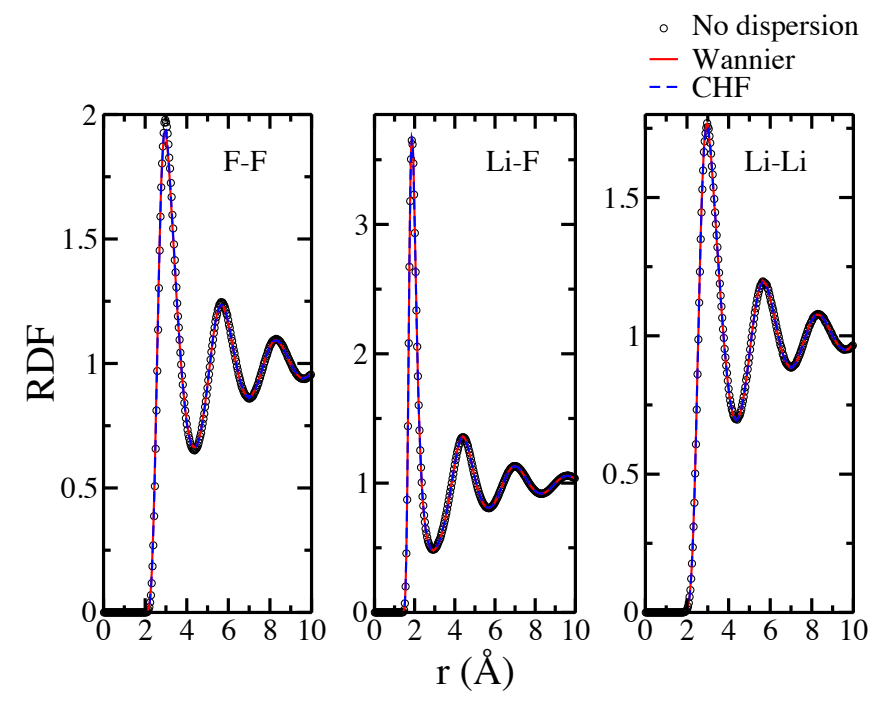

FIG. 2: Radial distribution functions in liquid LiF. Simulations were performed at $1200 \mathrm{~K}$, at the experimental density $\rho_{\text {exp }}=1.77 \mathrm{~g} \mathrm{~cm}^{-3}$.

$\mathrm{O}-\mathrm{H}$ radial distribution functions and the diffusion coefficients were shown to vary substantially. This is not the case for LiF: as can be seen in Figure 2 no difference is observed for any of the three partial radial distribution functions. This is because in such a simple molten salt, the structure mostly arises from a competition between the Coulombic interaction, which induces strong charge ordering, and the overlap-repulsion which sets the firstneighbour distance. The dispersion term is much weaker than those two and therefore does not change the structure.

The diffusion coefficients are extracted from molecular dynamics simulations using Einstein's relation, i.e. from the long-time slope of mean squared displacement:

$$
D(\alpha)=\lim _{t \rightarrow \infty} \frac{1}{6 N_{\alpha} t} \sum_{i \in \alpha, i=1}^{N_{\alpha}}\left\langle\left|\mathbf{r}_{i}(t)-\mathbf{r}_{i}(0)\right|^{2}\right\rangle
$$

where $N_{\alpha}$ is the total number of atoms of type $\alpha$, and $\mathbf{r}_{i}(t)$ is the position of atom $i$ of type $\alpha$ at time $t$. We obtain similar values for the three simulation setups, i.e. $7.4 \cdot 10^{-5}$ and $10.0 \cdot 10^{-5} \mathrm{~cm}^{2} \mathrm{~s}^{-1}$ for $\mathrm{F}^{-}$and $\mathrm{Li}^{+}$ions, respectively. Note that these values are in excellent agreement with the experimental ones [52, 53. Again, this situation is very different from that observed in liquid water, in which a diffusion coefficient two to three times larger was obtained when dispersion effects were included in DFT-based molecular dynamics [6] (although these numbers might be mitigated due to the use of short simulation times). In conclusion, due to the predominance of Coulombic and repulsion interactions, in contrast with the case of water, dispersion interaction does not play a major role on the physico-chemical properties of molten 


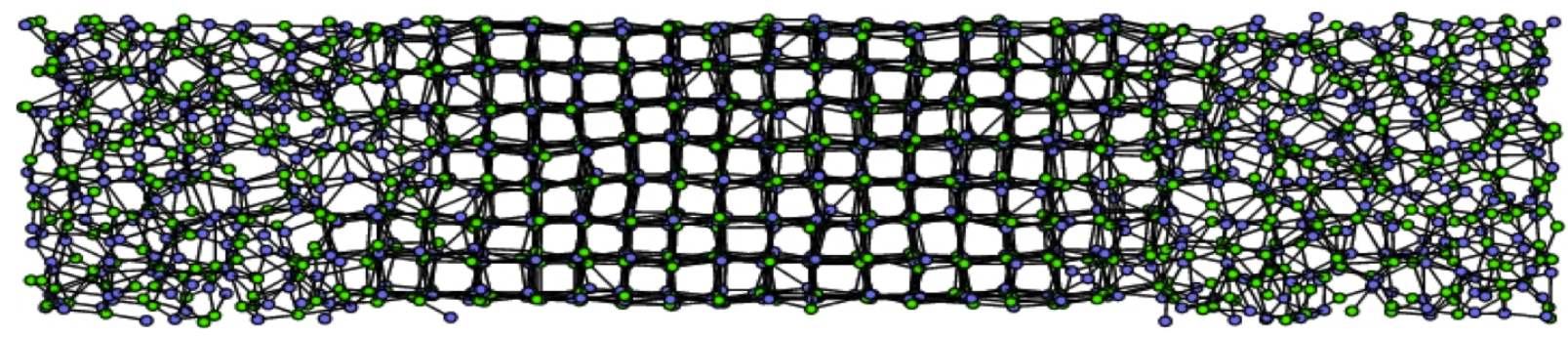

FIG. 3: Illustration of the simulation cell used to determine the $\mathrm{LiF}$ melting point (green: $\mathrm{F}^{-}$, blue: $\mathrm{Li}^{+}$).

$\mathrm{LiF}$ at a given density.

\section{Solid-liquid interface}

The melting point is usually considered as a very stringent test of a force-field, since it depends on the relative free energies of the crystal and liquid phases. The melting point can be determined by creating a cell containing the solid and liquid at coexistence. The interface has to be constructed by combining two separate bulk simulations of the crystalline and molten phases each equilibrated at the same pressure and the estimated melting temperature [47, 54]. The cell parameters for the liquid phase have to match those of the crystalline simulation. The two cells are then placed in a supercell, with a large aspect ratio, as shown on Figure 3. A first estimation of the melting point is obtained via short simulations performed in the $N V E$ ensemble, before longer simulations are performed in the NPT ensemble. In the first stage, the system is run for a short period of time (1 ps) in a constant volume simulation with the thermostat set at the estimated coexistence temperature (or by regular velocity rescaling) to remove excess energy caused by bringing the solid and liquid together. Great care must be taken during these initial stages to ensure that one or other phase is not destroyed. Once the excessive relaxation energy has been removed, the system can be run on for a further $50 \mathrm{ps}$ in a $N V E$ simulation to ensure complete equilibration. We then perform a 50 ps production run, again in the $N V E$ ensemble, during which we determine the average temperature and pressure. Other $(T, P)$ coexistence points can then be sampled by performing additional simulations where the initial kinetic energy of the system is rescaled to a value above that of coexistence and then the system is allowed to re-equilibrate in an NVE simulation [47.

The variation of $P$ versus $T$ at coexistence is shown on Figure 4 for the three sets of dispersion parameters. At $P=1 \mathrm{~atm}$, we obtain approximate melting temperatures of $980 \mathrm{~K}, 1115 \mathrm{~K}$ and $1170 \mathrm{~K}$ for the case with null dispersion and with the CHF and Wannier parameters, respectively. We then perform long simulations in the

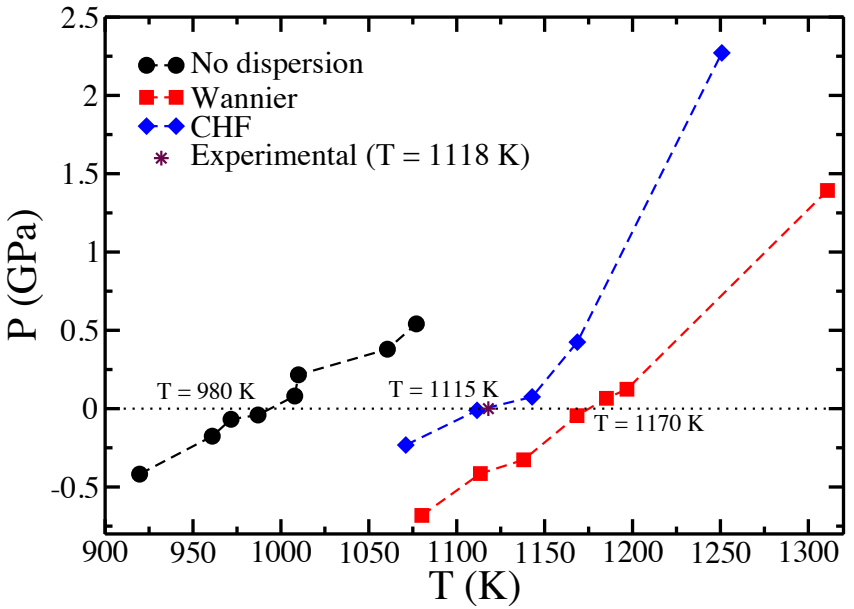

FIG. 4: $\quad(P, T)$ plots for $\mathrm{LiF}$ at the solid-liquid coexistence for the three sets of dispersion parameters.

NPT ensemble, where the target pressure is set to $1 \mathrm{~atm}$ while several target temperatures close to the estimated $T_{m}$ are studied.

We sample the potential energy and the cell length along the $z$ direction during these NPT simulations. The results are shown on Figure 5. Both quantities remain constant only when the liquid and solid are coexisting [45]. We extract refined melting temperatures of $1000 \mathrm{~K}, 1108 \mathrm{~K}$ and $1170 \mathrm{~K}$ for the case with null dispersion and with the CHF and Wannier parameters, respectively. This trend is easily understood by the fact that increasing the dispersion effects will result in a stabilization of the most condensed phase, i.e. the solid, with respect to the less condensed phase. Nevertheless, the observed differences are large (up to $>100 \mathrm{~K}$ ) despite the fact that no important differences were observed for the single phase structural properties. The CHF case provides a very accurate estimate of the melting point since the experimental value is $1118 \mathrm{~K}$. This result is somewhat surprising since the model using the Wannier dispersion parameters provided the best estimate of the densities of both the solid and liquid phases, again showing the difficulty of building a force field of perfect accuracy for all 


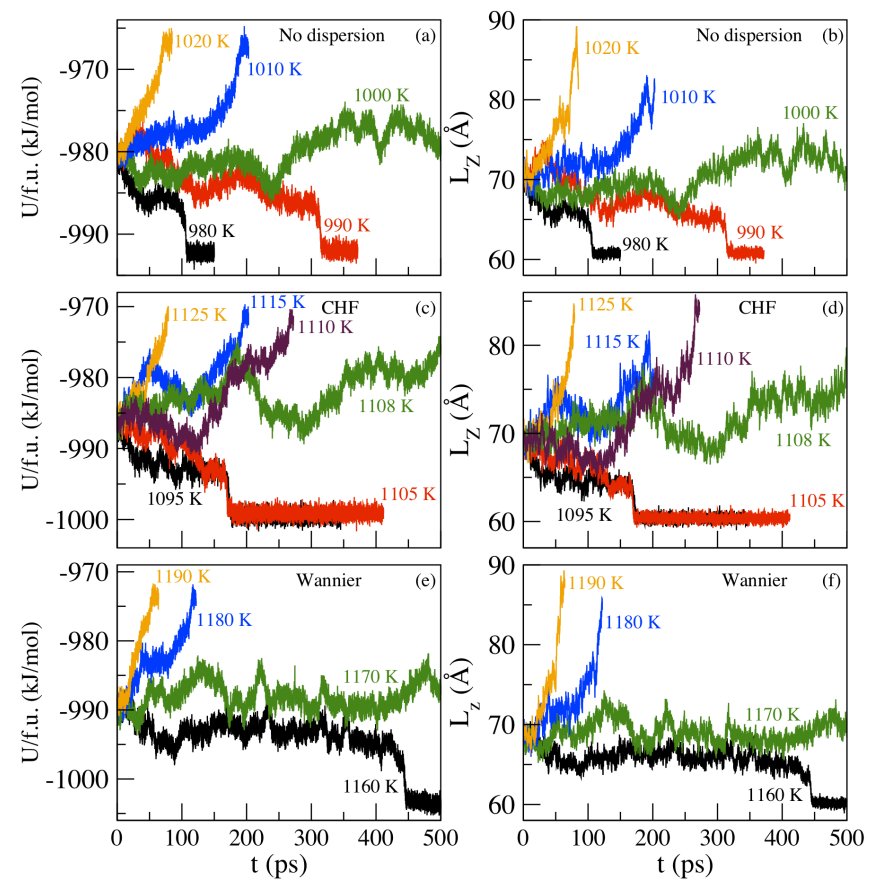

FIG. 5: Variation of the potential energy (left) and cell length along the $z$ axis (right) at several temperatures during NPT simulations performed at several temperatures.

the properties, even if it includes polarization effects.

\section{CONCLUSIONS}

In conclusion, we have shown in this paper that, although it brings a tiny contribution to the total energy, the dispersion interaction has important effects on the properties of $\mathrm{LiF}$. On the one hand, these effects are not observed in fixed volume simulations of the liquid, for which the structure and the dynamics are independent of the $C_{6}$ and $C_{8}$ terms. On the other hand, the predicted equilibrium densities are affected in both the liquid and solid phases: an underestimation of the experimental data by as much as $15 \%$ is observed when dispersion effects are omitted. But the strongest differences are obtained for the melting point, a quantity which reflects the free energy difference between the solid and liquid phase. This means that as soon as free energyrelated quantities have to be evaluated, special attention must be paid to using a correct parameterization of the dispersion coefficients.

To carry out this parameterization on a purely firstprinciples basis, which is what we have attempted here, is not straightforward. Here we have tested two sets of parameters. The first one was extracted from condensedphase Coupled Hartree Fock calculations 21, while the second one was obtained from the determination of the Maximally Localized Wannier Functions in a simple con- densed phase DFT calculation [16, 22. Both of them provide reasonably good predictions for the densities and the melting points, but more systematic calculations on a series of materials would be needed for identifiying the better parameterization procedure. It is to be expected that the developments which are currently being made for treating accurately dispersion interactions in DFT calculations [3 5] will provide useful routes.

\section{Acknowledgement}

The work has been performed under the HPCEUROPA2 project (project number: 228398) with the support of the European Commission - Capacities Area - Research Infrastructures.

\section{References}

[1] London F 1937 The general theory of molecular forces $T$. Faraday Soc. 33 8-26

[2] van der Waals J D 1910 The equation of state for gases and liquids Nobel Lectures in Physics 254-65

[3] Vydrov O A and van Voorhis T 2010 Dispersion interactions from a local polarizability model Phys. Rev. A $\mathbf{8 1}$ 062708

[4] Vydrov O A and van Voorhis T 2010 Nonlocal van der Waals density functional: The simpler the better $J$. Chem. Phys. 133244103

[5] Grimme S 2004 Accurate description of van der Waals complexes by density functional theory including empirical corrections J. Comput. Chem. 25 1463-73

[6] Lin I C, Seitsonen A P, Coutinho-Neto M D, Tavernelli I and Rothlisberger U 2009 Importance of van der Waals interactions in liquid water J. Phys. Chem. B 113 112731

[7] Jonchiere R, Seitsonen A P, Ferlat G, Saitta A M, and Vuilleumier R 2011 Van der Waals effects in ab initio water at ambient and supercritical conditions J. Chem. Phys. 135154503

[8] Schmidt J, VandeVondele J, Kuo I F W, Sebastiani D, Siepmann J I, Hutter J and Mundy C J 2009. Isobaricisothermal molecular dynamics simulations utilizing density functional theory: an assessment of the structure and density of water at near-ambient conditions J. Phys. Chem. B 113 11959-64

[9] Allen J P, Scanlon D O, Parker S C and Watson G W 2011 Tin monoxide: Structural prediction from first principles calculations with Van der waals corrections. $J$. Phys. Chem. C 115 19916-24

[10] Salanne M and Madden P A 2011 Polarization effects in ionic solids and melts Mol. Phys. 109 2299-315

[11] Grimme S, Hujo W and Kirchner B 2012 Performance of dispersion-corrected density functional theory for the interactions in ionic liquids Phys. Chem. Chem. Phys. 13 4875-83 
[12] Malberg F, Pensado A S and Kirchner B 2012 The bulk and the gas phase of 1-ethyl-3-methylimidazolium ethylsulfate: dispersion interaction makes the difference Phys. Chem. Chem. Phys. 14 12079-82

[13] Pensado A S, Brehm M, Thar J, Seitsonen A P and Kirchner B 2012 Effect of dispersion on the structure and dynamics of the ionic liquid 1-ethyl-3-methylimidazolium thiocyanate. ChemPhysChem 13 1845-53

[14] Aguado A, Bernasconi L, Jahn A and Madden P A 2003 Multipoles and interaction potentials in ionic materials from planewave-DFT calculations. Faraday Discuss. 124 171-84

[15] Madden P A, Heaton R J, Aguado A and Jahn S 2006 From first-principles to material properties J. Mol. Struct.: THEOCHEM 771 9-18

[16] Rotenberg B, Salanne M, Simon C and Vuilleumier R 2010 From localized orbitals to material properties: building classical force fields for nonmetallic condensed matter systems Phys. Rev. Lett. 104138301

[17] Salanne M, Rotenberg B, Simon C, Jahn S, Vuilleumier $\mathrm{R}$ and Madden P A 2012 Including many-body effects in models for ionic liquids Theor. Chem. Acc. 1311143

[18] Heaton R J, Madden P A, Clark S J and Jahn S 2006 Condensed phase ionic polarizabilities from plane wave density functional theory calculations. J. Chem. Phys. 125144104

[19] Salanne M, Vuilleumier R, Madden P A, Simon C, Turq P and Guillot B 2008 Polarizabilities of individual molecules and ions in liquids from first principles $J$. Phys.: Condens. Matter 20494207

[20] Molina J J, Lectez S, Tazi S, Salanne M, Dufrêche J.F., Roques J, Simoni E, Madden P A and Turq P. 2011 Ions in solutions: Determining their polarizabilities from first-principles J. Chem. Phys. 134014511

[21] Fowler P W, Knowles P J and Pyper N C 1985 Calculations of 2-body and 3-body dispersion coefficients for ions in crystals Mol. Phys. 56 83-95

[22] Silvestrelli P L 2008 Van der Waals interactions in DFT made easy by Wannier functions Phys. Rev. Lett. 100 053002

[23] Marzari N and Vanderbilt D 1997 Maximally localized generalized Wannier functions for composite energy bands Phys. Rev. B 56 12847-65

[24] Marzari N, Mostofi A A, Yates J R, Souza I and Vanderbilt D 2012 Maximally localized Wannier functions: Theory and applications Rev. Mod. Phys. 84 1419-75

[25] Madden P A and Wilson M 1996 "Covalent" effects in "ionic" systems" Chem. Soc. Rev. 25 339-50

[26] Tang K T and Toennies J P 1984 An improved simple model for the van der Waals potential based on universal damping functions for the dispersion coefficients $J$. Chem. Phys. 80 3726-41

[27] Stone A J 1996 Theory of intermolecular forces (Oxford University Press, Oxford)

[28] Fowler P W and Madden P A 1985 Fluctuating dipoles and polarizabilities in ionic materials: calculations on $\mathrm{LiF}$ Phys. Rev. B 31 5443-55

[29] Domene C, Fowler P W, Wilson M and Madden P A 2002 A transferable representation of the induced multipoles in ionic crystals Mol. Phys. $1003847-65$

[30] J̈emmer P, Wilson M, Madden P A and Fowler P W 1999 Dipole and quadrupole polarization in ionic systems: ab initio studies J. Chem. Phys. 111 2038-49

[31] Press W H, Flannery B P, Teukolsky S A and Vetterling
W T 1992 Numerical Recipes, 2nd editon (Cambridge University Press, Cambrdige)

[32] CP2K http://www.cp2k.org/

[33] Chen Z M, Cagin T and Goddard W A III 1997 Fast Ewald sums for general van der Waals potentials J. Comput. Chem. 18 1365-70

[34] Burbano M, Marrocchelli D, Yildiz B, Tuller H L, Norberg S T, Hull S, Madden PA and Watson G W 2011 A dipole polarizable potential for reduced and doped $\mathrm{CeO}_{2}$ obtained from first principles J. Phys.: Condens. Matter 23255402

[35] Marrocchelli D, Bishop S R, Tuller H L and Yildiz B 2012 Understanding chemical expansion in non-stoichiometric oxides: Ceria and zirconia case studies Adv. Func. Mat. 22 1958-65

[36] Salanne M, Marrocchelli D and Watson G W 2012 Cooperative mechanism for the diffusion of $\mathrm{Li}^{+}$ions in $\mathrm{LiMgSO}_{4} \mathrm{~F}$ J. Phys. Chem. C 116 18618-25

[37] Tangney P and Scandolo S 2002 An ab initio parametrized interatomic force field for silica J. Chem. Phys. 117 8898-904

[38] Han X J, Berqvist L, Dederichs P H, Müller-Krumbhaar H, Christie J K, Scandolo S and Tangney P 2010 Polarizable interatomic force field for $\mathrm{TiO}_{2}$ parametrized using density functional theory Phys. Rev. B 81134108

[39] Perdew J P, Burke K and Ernzerhof M 1996 Generalized gradient approximation made simple Phys. Rev. Lett. $7 \mathbf{7}$ 3865-68

[40] Martyna G J, Tobias D J and Klein M L 1994 Constant pressure molecular dynamics algorithms J. Chem. Phys. $1014177-89$

[41] Janz G J, Dampier F W, Lakshminarayanan G R, Lorenz P K and Tomkins R P T 1968 Molten Salts: Volume 1, Electrical Conductance, Density, and Viscosity Data (National Standard Reference Data Series - National Bureau of Standards 15)

[42] Nosé S 1984 A molecular dynamics method for simulations in the canonical ensemble Mol. Phys. 52 255-68

[43] Hoover W G 1985 Canonical dynamics: equilibrium phase-space distributions Phys. Rev. A 31 1695-97

[44] Espinosa J R, Sanz E, Valeriani C and Vega C 2013 On fluid-solid direct coexistence simulations: The pseudohard sphere model J. Chem. Phys. 139144502

[45] Conde M M, Gonzalez M A, Abascal J L F and Vega C 2013 Determining the phase diagram of water from direct coexistence simulations: The phase diagram of the TIP4P/2005 model revisited J. Chem. Phys. 139154505

[46] Hong Q J and van der Walle A 2013 Solid-liquid coexistence in small systems: A statistical method to calculate melting temperatures J. Chem. Phys. 139094114

[47] Lanning O J, Shellswell A and Madden P A 2004 Solidliquid coexistence in ionic systems and the properties of the interface Mol. Phys. 102 839-55

[48] Salanne M, Simon C, Turq P and Madden P A 2009 Heat-transport properties of molten fluorides: determination from first-principles J. Fluorine Chem. 130 38-44

[49] Dewan L C, Simon C, Madden P A, Hobbs L W and Salanne M 2013 Molecular dynamics simulation of the thermodynamic and transport properties of the molten salt fast reactor fuel $\mathrm{LiF}_{-} \mathrm{ThF}_{4}$ J. Nucl. Mater. $434322-$ 27

[50] J̈emmer P, Fowler P W, Wilson M and Madden P A 1998 Environmental effects on anion polarizability: variation with lattice parameter and coordination number J. Phys. 
Chem. A 102 8377-85

[51] Wilson M, Madden P A, J̈emmer P, Fowler P W, Batana A, Bruno J, Munn R W and Monard M C 1999 Models of environmental effects on anion polarizability Mol. Phys. 96 1457-67

[52] Sarou-Kanian V, Rollet A-L, Salanne M, Simon C, Bessada C and Madden P A 2009 Diffusion coefficients and local structure in basic molten fluorides: in situ NMR measurements and molecular dynamics simula- tions. Phys. Chem. Chem. Phys. 11 11501-6

[53] Levesque M, Sarou-Kanian V, Salanne M, Gobet M, Groult H, Bessada C and Rollet A-L 2013 Structure and dynamics in yttrium-based molten rare earth alkali fluorides J. Chem. Phys. 138184503

[54] Aguado A and Madden P A 2005 New insights into the melting behavior of $\mathrm{MgO}$ from molecular dynamics simulations: the importance of premelting effects Phys. Rev. Lett. 94068501 